\title{
INVESTIGACIÓN Y ACCIÓN PARTICIPATIVA EN LA ORGANIZACIÓN DE PEQUEÑOS PRODUCTORES
}

\author{
Research and participatory action in the organization of small-scale producers
}

Uguet Vaquer Piloni, Jaime P.

Gdor. Ferre 2842 AER INTA Virasoro. E-mail: uguet.pablo@inta.gob.ar

\begin{abstract}
RESUMEN
Este trabajo fue parte de un proyecto de PROFEDER (Programa Federal de Apoyo al Desarrollo Rural) de gestión INTA (Instituto Nacional de Tecnología Agropecuaria) con fondos de Desarrollo Social. Se aplicó Metodología de Acción Participativa (investigación y acción participativa) con un grupo de pequeños productores del departamento de General Alvear Corrientes, con los cuales se logró avance en cuanto a lo organizacional. Estos resultados fueron construidos desde los acuerdos logrados y discutidos desde las bases gracias a la aplicación de esta metodología, la cual ordena a través de técnicas la expresión de las ideas. Se logró establecer las bases que siguen atendiendo a las necesidades de los productores, desarrollando sus actividades de manera conjunta, planificando y gestionando las herramientas que necesiten como grupo.
\end{abstract}

Palabras Clave: Metodología, acuerdos, organizacional.

\begin{abstract}
This work was part of a PROFEDER (Federal Program for Rural Development Support) project managed by INTA (National Institute of Agricultural Technology) with Social Development funds. Participatory Action Methodology was carried out by a group of small-size producers in General Alvear in Corrientes whose progress was based on its organization. The results obtained were reached due to consensus agreement among the participants who applied the Participatory Action Methodology to express their own ideas. Bases were achieved to overcome producers' needs; developing their cooperative work, planning and managing the tools they need to work as a group.
\end{abstract}

Key words: Methodology, agreements, organizational.

\section{INTRODUCCIÓN}

Empezamos el trabajo reconociendo que todo proyecto tiene bases teóricas e ideológicas que lo sustentan, aunque muchas veces sean inconscientes. Cuando nos acercamos a cualquier realidad, para conocer algo sobre ella, muchas veces ya tenemos razones por lo que lo hacemos, razones tal vez no comprendidas suficientemente, pero no por ello menos reales. La aplicación de Metodologías de Acción Participativa (investigación y acción participativa), nos ayuda a cuestionar las labores que hacemos normalmente. En conjunto, todos estos métodos, se caracterizan por una secuencia común de actuación (Guzmán et al., 1998). La primera fase se centra en el análisis y diagnóstico de los problemas y de las prácticas del agricultor. Incluye una evaluación rápida y eficiente económicamente, generalmente a través de la técnica de Diagnóstico Rural Rápido, realizado por un equipo multidisciplinar. Una segunda etapa de planificación experimental e investigación en la finca con el agricultor, en la que éste aporta sus opiniones y, por último, la fase de evaluación por adopción de los agricultores (Guzmán et al 1998).

Recibido: 09/jun/2020. Aceptado: 01/sep/2020 
Un grupo de pequeños productores del Departamento General Alvear (Corrientes) manifestaba con algunas acciones, la falta de ejercicio en el trabajo conjunto y la organización, a pesar de encontrarse dentro de una asociación, Como lo menciona Geilfus 2002, la asociación voluntaria o acción colectiva, posee un formidable potencial transformador si es aprovechado adecuadamente. Por esta razón vimos la posibilidad de implementar con el proyecto trabajos de Acción e Investigación Participativa y así solucionar esta problemática. Conocer lo que llamamos "realidad de los productores", que puede ser tan normal para algunos actores, hasta incluso su razón de ser; para otros puede resultar una cuestión intrínseca y difícil de abordar. Explicitar, discutir, poner en duda estas bases, bajo la aplicación de metodología, fue el primer desafío al emprender este proyecto. Estos cuestionamientos nos llevaron a la construcción de principios mínimos, consensuados y explicitados que sustentaron todo el trabajo, tanto su para qué, sus por qué y su forma de llevarlo a cabo.

El objetivo del proyecto fue fortalecer la organización de los a través de técnicas de investigación y acción participativa.

\section{METODOLOGÍA}

El trabajo se desarrolló en el departamento General Alvear ubicado al este de la provincia de Corrientes, en la desembocadura del Río Aguapey, a orillas del Río Uruguay. La ciudad de Alvear se encuentra sobre la Ruta Nacional 14 a 440 kilómetros de la Ciudad de Corrientes. Según datos arrojados por el Censo poblacional INDEC 2010 su población era de 8.001 habitantes.

Los parajes rurales donde se asientan los productores agrícolas se radican en Arroyo Méndez; Pancho Cué; Palmitas; Santa Rosa y Pirayú. Los actores con los que se trabajó en este proyecto, son pequeños productores que se encontraban dentro de la "Asociación de pequeños productores Pancho Cué Palmita", institución que se encontraba dentro de una mesa interinstitucional (resultados de un proyecto PROFEDER anterior). La Asociación al inicio de este proyecto, comenzaba la organización, ya que no tenía más de un año de formación.

Se aplicaron metodologías de acción participativas, que se basaron en 80 herramientas para el desarrollo participativo: diagnóstico, planificación, monitoreo, evaluación (Geifus, 2002).

- Técnicas de diálogo, observación y dinámica de grupo: diálogo semiestructurado, diálogo con informantes clave y lluvia de ideas.

- Diagnóstico participativo de aspectos generales de la comunidad (aspectos sociales): Perfil de grupo.

- Análisis organizacional / institucional: diagrama de Venn, mapa de servicios y oportunidades.

- Diagnóstico participativo de sistemas de producción: Clasificación preliminar de finca (según acceso a los recursos), flujograma de actividades y uso del tiempo.

- Análisis de problemas y soluciones: Árbol de problemas: diagrama de causas y efectos, matriz de priorización de problemas, análisis FODA y análisis de pro y contra (juego de sí señor - no señor).

- Planificación: Mapa de ordenamiento de finca y plan de la finca.

Mediante la aplicación de esta metodología, se buscó plantear alternativas que se fundamentan en la plena participación de los productores, y que con eso se lleve a una práctica consciente y transformadora de su realidad.

También se usaron como insumos metodologías de extensión tradicionales, visitas a los distintos referentes de la zona (charlas personales) y organización de reuniones con productores a fin de identificar y sociabilizar con los actores que trabajarían en el proyecto. Se recurrió al uso del Registro Nacional Sanitario de Productores Agropecuarios (RENSPA) de SENASA para tener números reales de las unidades productivas y situación de los actores.

Con este proyecto de la cartera PROFEDER y como parte de INTA, se acudió a la mesa interinstitucional de la ciudad de Alvear, formada por el Municipio, la escuela Agrotécnica, Plan Ganadero Provincial, SENASA, FUCOSA, Asociaciones de pequeños productores y Policía Rural (PRIAR). Esta mesa fue el resultado de un proyecto de desarrollo anterior, en la que se discutieron problemáticas del sector desde la visión de las instituciones del medio y fue parte de la gestión de soluciones para el sector con el que trabajaba el proyecto. 
Uguet Vaquer Piloni, J.P.: Investigación y acción participativa.

\section{RESULTADOS}

\section{Técnicas de diálogo, observación y dinámica de grupo}

Usando la técnica de Diálogo Semiestructurado y Diálogo con Informantes Clave se buscó recolectar información general sobre los productores.

Con esta técnica se buscó evitar algunos de los efectos negativos de los cuestionarios formales, como ser temas cerrados, la falta de diálogo o la falta de adecuación a las interpretaciones de los actores.

Luego se trabajó con un grupo reducido de productores directamente involucrado en la asociación. Se utilizó la técnica de lluvia de ideas, con el objetivo de obtener información pertinente, en forma rápida, recolectando todas las ideas y conocimientos de la gente, para ser usados en los sucesivos talleres.

Se identificó que el $40 \%$ de los productores participantes tenían menos de 50 ha y problemas de tenencia de la tierra por ejemplo por sucesiones inconclusas o utilización de chacras solo por separación de palabra entre familiares. Esto acarrea problemas a la hora de tramitar préstamos o incentivos que tienen como requisito tener los documentos de la tenencia de los predios.

El productor tipo de la zona es un pequeño granjero que tiene diversidad de producciones, con un fuerte arraigo en la ganadería como ahorro. Esta situación lleva a que sus chacras se encuentren con el manejo actual al límite de lo sustentable, siendo muy frágiles a las circunstancias del clima y ámbito comercial.

\section{Diagnóstico participativo aspectos generales de la comunidad (aspectos sociales):}

Se utilizaron distintas herramientas como ser el taller de perfil de grupo (Figura 1), buscando definir conjuntamente las características del grupo de participantes, en relación con las actividades examinadas. Con esto se buscó conocer, en sentido general, las características socio-económicas, productivas cualitativas y cuantitativas de lo obtenido en sus chacras.

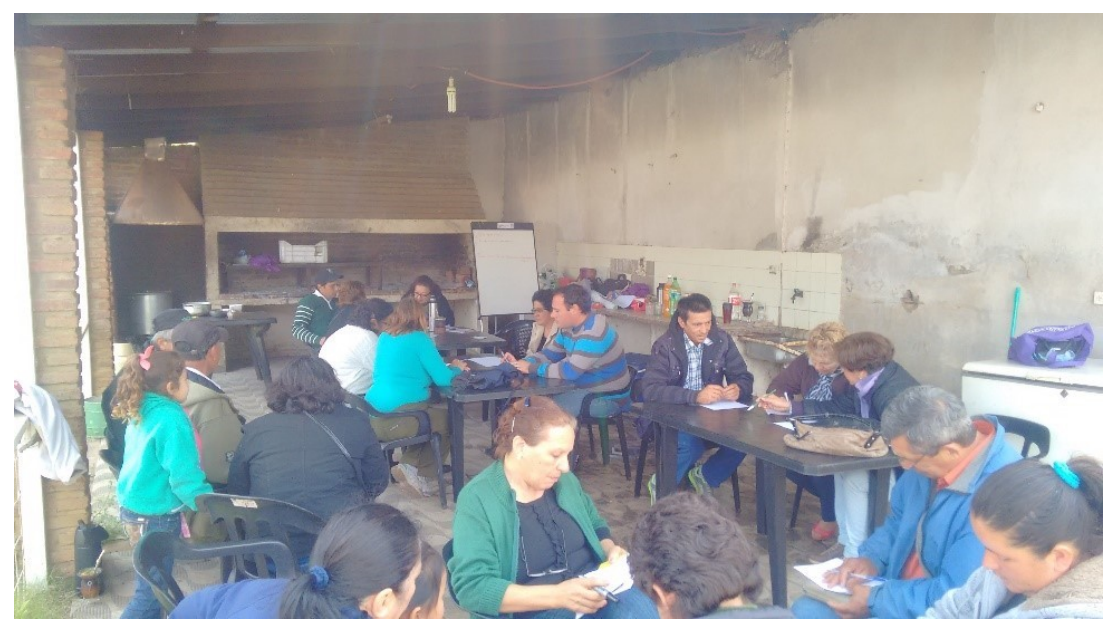

Figura 1. Productores trabajando en grupos en Taller participativo Perfil de Grupo, cumpliendo con parte de las consignas asignadas.

Si bien la mayoría tenia ganadería como actividad, el perfil general del grupo mostró que el $90 \%$ se asentaba sobre actividades hortícolas y en menor medida pecuarias (venta de huevos y gallinas), usando la ganadería para consumo propio o ventas casuales en caso de necesidades particulares (ingresos extraordinarios). Esta técnica se utilizó con los productores que trabajaban en la formación de Feria Franca. Ellos identificaron que más del 70\% de los productos que ofrecían en sus eventos de ventas, pertenecían a panificados, productos de los cuales ellos no podían influir en gran parte de la formación de los precios y por ende de sus ganancias. A partir de esto se evidenció la importancia de trabajar en el desarrollo y ajuste de la producción primaria y en el agregado de valor en la venta de estos en la Feria Franca.

Como parte del diagnóstico se evaluaron los canales comerciales. El 80\% de los productores vendía sus productos puerta a puerta invirtiendo mucho tiempo en la comercialización y descuidando lo productivo. 
La realización del taller de mapa de servicios y oportunidades: ayudó a establecer una representación gráfica de los servicios comunitarios (escuela, salud, transporte, etc.) y oportunidades de comercialización, servicios, utilizados por los productores participantes. Como resultado se obtuvo que el 100\% de los productores cuentan con medios propios de movilidad (auto, motos, caballos) para llegar al pueblo y hacer sus ventas o trámites. Cuentan con una escuela primaria rural y una escuela agropecuaria para estudios secundarios que se encuentra en la localidad de Alvear. y que cuenta con un internado, de esta manera reduce los viajes de los alumnos.

\section{Diagnóstico participativo de sistemas de producción:}

Se realizó una clasificación preliminar de Finca según acceso a los recursos. Se analizó junto a los productores el nivel de sustentabilidad de las chacras, y de qué forma el acceso a los recursos determinan, según ellos, estos niveles. De aquí se desprendió que todos los productores participantes justificaban sus problemas productivos a la falta de acceso a recursos como semillas, alimento para el ganado, tiempo operativo entre otros.

Aplicando la herramienta de Flujograma de Actividades, se representó en forma esquemática, el flujo de eventos y decisiones necesario para llevar a cabo una actividad productiva determinada, como ser producción de lechuga o producción de huevos. Este diagrama se utilizó para discutir la problemática productiva de los emprendimientos y analizar la complejidad de los conocimientos necesarios para llevarlos a cabo correctamente. También se analizó el uso del tiempo tanto para los hombres como las mujeres y sobre el aporte real de la mujer en las actividades de la explotación familiar; sirviendo para disipar mitos sobre el papel limitado de las mujeres. Con esta herramienta sencilla, a través de diagramas se pusieron de manifiesto algunas cuestiones que en los quehaceres diarios los productores no visibilizan.

\section{Análisis de problemas y soluciones:}

Con la aplicación de las distintas técnicas se buscó profundizar el ejercicio de identificar problemas en el sentido analítico. Esto ayudó a los técnicos y a los productores a entender mejor la problemática, y distinguir entre causas y efectos.

A pesar de ser bastante complejo, con el tiempo y la ejercitación se puede llegar a definir cuáles son las causas de los principales problemas y priorizarlos para trabajar en su solución.

\section{Planificación:}

Esta etapa es crucial como dice Hernández (2010), el actuar sin planificación, sin objetivos claros, un hacer por hacer, no cambia nada en profundidad. Esto pasa cuando hay muy buena voluntad, mucha discrepancia, pero sin planificación en el sentido de tener un "para qué" claro y sistematizado que permita hilar unos por qué y unos qué hacer y asimismo una evaluación.

En este sentido aplicamos la herramienta de Mapa de ordenamiento de finca, que busca producir un mapa que representa un horizonte u objetivo final imaginado por la familia, en cuanto al orden de su chacra. Los productores esquematizaron un mapa de la chacra donde ubicaban sus producciones. En este caso se realizó una proyección o "visión" de las chacras, a tres años, que era la duración del proyecto. Esto no fue un plan realizado por el técnico, sino un trabajo de la familia de cada chacra. Sería un documento básico para visualizar la planificación y cambios en el manejo de recursos a nivel de sus chacras.

Luego se avanzó con el Plan de la Finca, buscando definir en un plan las opciones que se ofrecían para desarrollar en cada chacra y las acciones necesarias para llevarlo adelante. Este plan se acordó entre los productores con la facilitación del técnico extensionista (responsable del proyecto). Este trabajo no buscó ser un plan ideal, sino un compromiso sobre acciones factibles a corto y mediano plazo, para acercarse a la visión plasmada en el mapa de ordenamiento de la chacra.

La planificación no es un proceso complejo, pero requiere pasos lógicos, el diagnóstico, análisis de los problemas, selección de opciones y elaboración del plan.

La implementación de los distintos talleres de metodología participativa nos ayudó a construir las bases y al desarrollo del proyecto, se buscó exponer capacidades colectivas e individuales de los participantes y así, aprovechar las oportunidades del medio para la mejora integral de los productores. 
Desde nuestro papel de facilitadores, aportamos ideas, viendo la integralidad de las situaciones, involucrándonos, reflexionando continuamente sobre nuestro quehacer y compartiendo experiencias propias y ajenas. Este ejercicio fundamentó la construcción, validación o transformación de prácticas productivas consideradas por los actores como normales.

Se impulsaron reuniones con instituciones y organizaciones y se analizaron líneas de trabajo y financiamiento que aportaron a la finalidad del proyecto que tenían los participantes.

Parte del trabajo institucional se facilitó por la existencia de una mesa interinstitucional. En la que se trabajó en líneas integrales donde cada participante aporta desde su lugar.

En el primer período del proyecto se avanzó, valiéndose de las capacidades innatas de algunos productores predispuestos, como demostradores de técnicas y alternativas sustentables de producción, "módulos demostrativos en chacras" (Figuras 2 y 3 ).

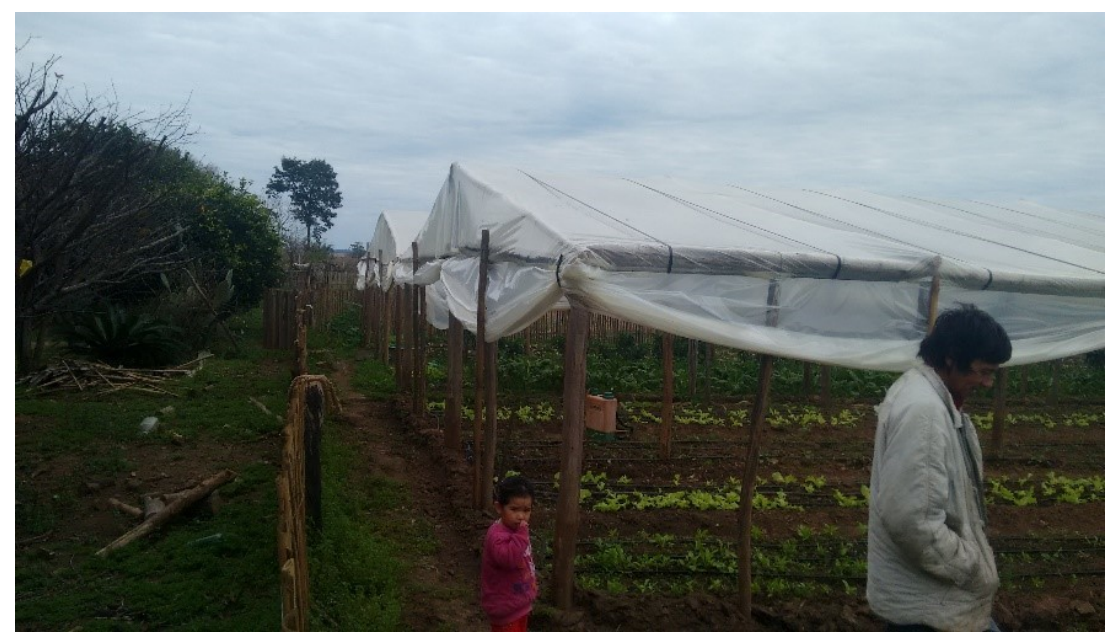

Figura 2. Productor de Paraje Palmita modulo demostrativo, tendaleros con producción hortícola (lechuga, cebolla de verdeo y perejil).

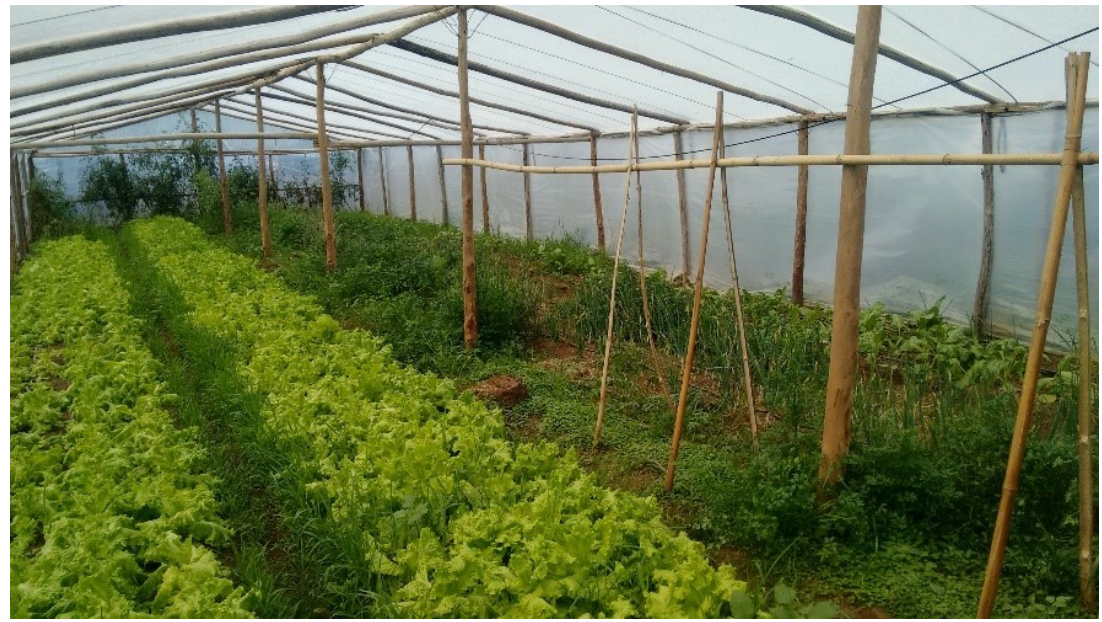

Figura 3. Modulo demostrativo Paraje Pancho Cué, tendaleros con producción hortícola (lechuga, acelga, tomate, cebolla de verdeo y perejil).

Una vez que los actores identificaron su perfil productivo, se siguió trabajando como vimos anteriormente en distintos talleres donde siempre se buscó que los productores manifiesten sus necesidades desde lo social y lo productivo, siempre en la base de una manifestación y acción voluntaria. Luego a través de las distintas herramientas se trabajó con acciones concretas para la obtención de los objetivos planteados. 
Al finalizar el proyecto, la Feria contaba con 10 feriantes permanentes, y otros ocasionales, todos parte de la Asociación y agrupados en la Asociación de Ferias provinciales (Figura 4).

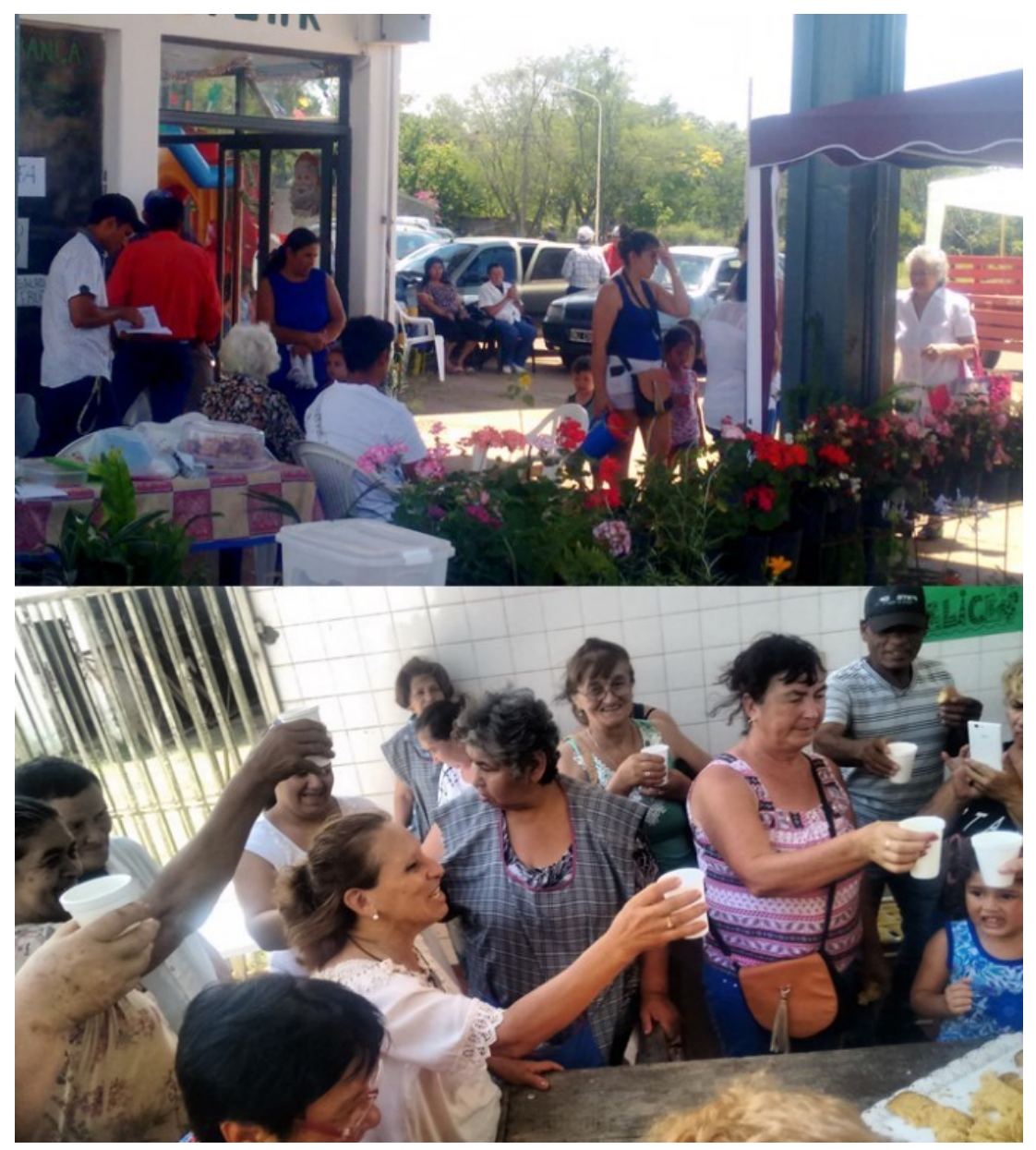

Figura 4. Encuentro de feria de fin de años 2015, feriantes de la Asociación de pequeños productores Pancho Cué Palmita y con la participación de Ferias de Virasoro y Santo Tome.

Con este trabajo se logró planificar y atender la preparación de suelos en un $80 \%$ de las chacras. La planificación de la producción estaba destinada por ejemplo a mandioca, cucurbitáceas, maíz para venta en fresco y pasturas perennes, siempre atendiendo las necesidades de la comunidad participante.

Con aporte de Fondos de PROFEDER (Programa Federal de Apoyo al Desarrollo Rural) la Asociación generó un Fondo Rotativo que fue destinado a la implementación y construcción de tendaleros plásticos con el fin de producir hortalizas frescas. La cuales eran parte fundamental en el fortalecimiento de los ingresos diarios de los productores involucrados.

Un punto central e importante en este trabajo es el compromiso de los actores quienes, realizando beneficios comunitarios desde su Asociación sumaron fondos a los recibidos mediante un proyecto de Agricultura Familiar e hicieron posible la compra de herramientas para la preparación de suelos.

Hasta la culminación de este proyecto organizacional, se construyeron 6 tendaleros de $20 \mathrm{~m} \times 50 \mathrm{~m}$, con productores que hasta ese momento no contaban con esa tecnología. Al participar de este fondo uno de los compromisos planteados fue, destinar parte de la producción a la venta en la "Feria Franca Alvear" que es parte de la Asociación.

\section{CONSIDERACIONES FINALES}

Con la forma participativa de trabajo se buscó dar respuestas a necesidades actuales. Se aportó desde el proyecto un pequeño grano de arena para mejorar la situación de los productores, viendo la totalidad de situaciones, comprometiéndonos y reflexionando continuamente sobre nuestro quehacer y compartiendo nuestras experiencias. 
Uguet Vaquer Piloni, J.P.: Investigación y acción participativa.

Fue un reto de superar barreras que se han ido creando artificialmente, viejas divisiones entre profesionales y productores, entre instituciones e incluso entre personas que responden a ciertos modelos de organización.

Este proyecto dejó sentadas las bases que siguen atendiendo las necesidades de los productores, quienes aún hoy continúan desarrollando sus actividades de manera conjunta, entendiendo que esto es posible por su propio compromiso.

Si bien estamos formados para medir la tarea de muchos proyectos y la labor de extensión, la generación de productos e indicadores que den cuenta del trabajo, el número de módulos demostrativos, cantidad de producción lograda, personas involucradas, no deberíamos perder de vista el proceso que nos deja un continuo aprendizaje. Con nuestras evoluciones internas y los cambios por los que trabajamos en Extensión se logran grandes y permanentes transformaciones, que muchas veces pasan desapercibidas.

Trabajar con esta metodología es una experiencia nueva e interesante, el desafío a futuro sería sistematizar los registros, así poder mostrar estos trabajos con mejor representación.

Por la formación técnica profesional de los ingenieros agrónomos, la utilización de metodologías sociales fue un gran desafío. Incursionar en herramientas de extensión que nos acercan a los productores y nos ayudan a desentramar lógicas muchas veces poco entendidas desde nuestra realidad, nos ayudó a generar bases que aportan al desarrollo de sectores productivos que cuentan con problemáticas estructurales diversas. Muchas veces el inicio de la solución está en mano de cambios sociales individuales y comunitarios en los cuales, la organización cumple un rol fundamental.

\section{BIBLIOGRAFIA}

Geilfus, F. (2002). 80 herramientas para el desarrollo participativo: diagnóstico, planificación, monitoreo, evaluación. Instituto Interamericano de Cooperación para la Agricultura (IICA).

Guzmán, G.C., Alonso Mielgo, A., Pouliquen, Y. y Sevilla Guzmán, E. (1998). Las Metodologías participativas de investigación: un aporte al desarrollo local endógeno. Actas del II Congreso de la Sociedad Española de Agricultura Ecológica. Agricultura Ecológica y Desarrollo Rural. Disponible en: https:// www.terceridad.net/sc3/Por_Tema/2_Metodo_IA_IP/Apoyo_2 $/ \operatorname{metdolog} \%$ EDas $\%$ 20participativas_X.pdf

Hernández, L. (2010). Antes de empezar con metodologías participativas. Cuadernos CIMAS Observatorio Internacional de Ciudadanía y Medio Ambiente Sostenible. Disponible en: https://www.redcimas.org/ wordpress/wp-content/uploads/2012/08/m_DHernandez_ANTES.pdf 